\title{
Conceptual Framework for Integration of Organisation Information Processing Theory and Activity Theory in Enterprise Systems Implementation
}

\author{
Clement Mayieko Nyandiere ${ }^{{ }^{*}}$, Faustin Kamuzora ${ }^{1}$, Ismail Ateya Lukandu ${ }^{1}$, \\ and Vincent Omwenga ${ }^{1}$
}

${ }^{1}$ Faculty of Information Technology, Strathmore University, P.O. Box 24601-00100 Nairobi, Kenya.

Research Article

Received: 7 June 2013

Accepted: 26 July 2013

Published: 05 October 2013

\begin{abstract}
Implementation processes of systems in organizations follow different strategies and one of these strategies is to understand the uncertainties associated with the integration of the new system into an existing system environment so as to limit any challenges that may arise during the system implementation. Systems are implemented to address specific organizational needs, that is, activities the organization engages in. This paper explores organization information processing theory (OIPT) and activity theory in enterprise resource planning (ERP) systems implementation. A framework for the ERP implementation has been developed from a conceptual model on the interaction of organizational culture and structure. The modified model incorporates the activity theory and the OIPT and provides a formal way of bringing on board various interplaying variables in the system implementation process, more so for ERP systems.

Keywords: OIPT, activity theory, information processing, enterprise systems, ERP implementation.

\section{Introduction}

Implementation processes of systems in organizations follow different strategies. One of these strategies is to understand the uncertainties associated with the integration of a new system into an existing system or structure. This is so as to limit any challenges that may arise during the system implementation. This is well appreciated through the organizational information processing theory (OIPT). The topic of OIPT has been discussed widely by different researchers, including $[1,2,3,4,5]$. The researchers have mainly discussed how OIPT helps address environmental considerations and uncertainties in systems implementation.
\end{abstract}

[3] uses the organizational information processing theory (OIPT) to explore uncertainties within

*Corresponding author: cnyandiere@gmail.com; 
an organization with the aim of finding a way of mitigating the challenges beforehand. In their application of the theory, [3] describe the uncertainties experienced by organizations as the difference between the amount of information required to perform the task and the amount of information already passed by the organization.

The sources of these uncertainties from the organizational perspective include technology, internal contexts and external contexts. Inherently these sources can be categorized into two; technological and environmental. The uncertainties can be associated with an absence of information, which may lead to acquisition of more data and may result in the inability to confidently assign probabilities about how environments will affect the success or failure of a decision-making task $[5,6]$. The challenges of reconciling these needs are the sources of inherent resistance to system implementation.

[2] indicates that environments are always the main sources of information uncertainty, which often impact the process of mission and influence, and the fit between organizational process and best practice brought about by enterprise resource planning (ERP) systems. In this paper we present a framework for the analysis of the environmental uncertainties and then bringing them on board during the system implementation indirectly through the development phase of the ERP so as to mitigate the challenges that may arise during system implementation by using the OIPT.

The documentation of this research write-up is organized as follows; in, section 2 we discuss a background to the study covering the organizational information processing theory (OIPT) and Activity Theory. In section 3 we present a model for the implementation of ERP incorporating concepts of activity theory and OIPT, addressing environmental uncertainties. We discuss the application of the model in a university set-up under section 4 . We present our conclusion and recommendation in section 5 .

\section{Background to OIPT and Activity Theory}

\subsection{Organizational Information Processing Theory and ERP Implementation}

Organizational information processing theory (OIPT) posits that resolving uncertainty is the central task in organizational design. Previous studies find a lot of factors that impede the connectivity of function and process that lead to the uncertainty, such as organizational misfit (i.e. data, process, use) [7], organizational resistance [8], adaptation problems (ERP adaptation, or process adaptation) [9], differentiation among sub-units [3], and organizational structure [10].

OIPT conceptualizes uncertainty as a lack of information about organisational tasks and the environment of operation [11]. The amount and types of uncertainty vary across organizations and among individual departments within organizations. Literature states that mechanisms such as hierarchical structures and standard operating procedures are appropriate when uncertainty is low, while computerized information systems and lateral relations are better choices in high uncertainty situations. Therefore, in order to prosper, the organization must match the appropriate mode(s) of coordination with its particular uncertainties [11].

[11] have further noted various sources or types of environmental uncertainty, including: the characteristics of the self-contained tasks that departments must execute (internal context), instability of the external environment, interdependence with other sub-units and differentiation 
among sub-units.

For the success in the implementation of the ERP, integration and standardization are considered as the most critical characteristics of ERP. These are closely related to the interdependencies and differentiation between sub-units of an organization as the main sources of uncertainties which are internal in context. Theory suggests that greater interdependence among organizational departments is associated with greater benefits from ERP; on the other hand, differentiation among organizational departments can lead to some significant ERP-related costs which are the most direct uncertainties in the implementation of ERPs [5,11].

In addressing the environmental uncertainty, we look at the complexity of the environment and dynamism or the frequency of changes to various environmental variables affecting systems implementation from the perspective of interdependencies and differentiation. [12] note that individuals operate in an environment of constant risk and uncertainty this influences the choices people make. Not only are individuals unaware of the outcomes that will result from strategic choices, but they are uncertain of the procedures of making choices and are also uncertain about their own preferences and procedures for choices they make [12].

In implementing ERPs, it helps to appreciate the conducted factors (CF) as well as environmental factors $(\mathrm{EF})$. [1] referencing various authors indicates that conducted factors $(\mathrm{CF})$ that influence ERP implementation success tend to be internal and include, among others, top management support, user support, project team member competence, project manager leadership skills, vendor support, consultants competence; level of system customization and data quality and postimplementation support for the ERP system. On the other hand, [5] and [6] have discussed environmental factors that influence organizational task environments as being among others: rapid technological changes, global competitors, unpredictability of customer taste, severe regulatory restrictions, shortage of labour or raw materials, relative lack of exploitable opportunities and resources.

[1] point out that conducted factors (CF) have the positive influence on coordination improvement and task efficiency while environmental factors (EF) have the positive influence on coordination improvement but negative influence on task efficiency, coordination improvement, and task efficiency have the positive influence on ERP benefits.

In the context of ERP implementation, there is need to remove any uncertainties that relate to ERP implementation. These uncertainties include: the system specifications, user requirements, budget (cost) estimates, time to be spent in the system implementation and how the system implementation process will affect normal operations of the business. The uncertainties need to be addressed at the time of systems planning and is significant, especially for large projects such as ERP implementation.

\subsubsection{Interdependence and differentiation factors in OIPT}

\subsubsection{Interdependence influence on ERP benefits}

Interdependence describes the degree to which organizational units exchange information or materials in order to complete tasks [13]. Interdependence increases the need for "mutual adjustment" and decreases the degree to which activities can be pre-planned. When interdependence is low, simple coordination modes, like standard operating procedures, suffice. 
However, high interdependence increases the need for a common formalized language in order to enable the exchange of information among departments. OIPT posits that the benefits of a highly integrated, standardized system, such as ERP, are influenced positively by the level of interdependence. That is, the higher the interdependence the more the benefits of the system to the users and organisational units.

Managing interdependence and improving the flow of information across departments is a major reason many firms have implemented ERP [13]. ERP facilitates departments to exchange information and materials. After all, data standards eliminate the burden of reconciling or translating information that is inconsistently defined across two or more departments. Data standards also do away with data ambiguity. ERP also improves the timeliness of information for decision making.

\subsubsection{Differentiation influence on ERP costs}

The costs of a standardized system, such as ERP, increase in proportion to the degree of departmental differentiation, which is the uniqueness of tasks, technologies, environment, goals, and standard operating procedures across the departments. According to a model developed by [11] when an integrated information system is implemented across a number of differentiated departments, two types of costs result: design costs and compromise costs.

Designing a single common system to accommodate the diverse needs of departments that differ greatly from one another is a difficult and expensive task, compared to designing a system for a collection of relatively homogeneous departments and units. These design costs include the time and money spent developing a common understanding across very different points of view, creating complex information system designs, and sometimes database structure redesign. On the other hand, when standardized systems are implemented across differentiated departments and units without such complex designs, some departments will experience compromise costs. These costs include decreased operational performance or decreased data relevance due to one or more departments having to use an information system that is not well-tailored to the specialised tasks that the department must execute. [9] found out that the fit of an organization's ERP system with its tasks, data, and related needs explains a significant amount of the company's ERP implementation success. Understanding these two factors, that is, interdependence and differentiation is critical for successful implementation of an ERP. In this paper, we explore these factors by applying the OIPT.

[4] note that OIPT identifies three important concepts, that is, information processing needs, information processing capability, and the fit between the two to obtain optimal performance. Information processing needs portend the user requirements, thus addressing the business area the system will be used to solve an organizational need. On the other hand the information processing capability addresses the system specifications and the features of the system that will make it effective for purposes of solving business problems it is expected to address.

[15] points out that the difficulty in ERP implementation in developed countries may be exacerbated by the claim that ERP embodies established ways of doing business thereby requiring organizations adopting ERP systems to change their business processes to conform to business practices inbuilt in ERP packages. Vendors argue that the adoption of these best practices makes the configuring of the software less costly and brings about improvement in the organization's processes. Furthermore, ERP systems are developed by vendors who draw on their existing 
sources of knowledge, resources and norms. These would include the developer organization's own business strategy and prevailing norms about what constitutes best practice. The challenge with this approach is that the ERP is developed without consideration of the implementation challenges that are environmental in nature. The lack of this consideration is considered to be the main source of resistance in the implementation of ERP by the users.

[4] further notes with increased information needs, the uncertainty can be dealt with by developing buffers to reduce the effect of the uncertainty, and by implementing structural mechanisms and information processing capability to enhance the information flow and thereby reduce uncertainty. Thus, the establishment of the likely uncertainties before the design of the ERP is important for the success of the implementation of the ERP.

Redesign of business processes in organizations and implementation of integrated information systems, such as ERPs, is expected to improve information flow and reduce uncertainty within organizational units. This strategy can also create better information flow between organizations to address the uncertainties in an organization. In this paper, we propose the consideration of the uncertainties during the redesigning stage by the application of OIPT to evaluate the associated interdependencies and differentiations. By applying the OIPT in the ERP implementation an organization needs not to re-organize itself but rather the ERP should fit into the organization, thus eliminating any resistance (by users- both management and workers) to the system implementation process.

\subsection{Activity Theory and ERP Implementation}

Activity theory informs systems design through the identification of the actions and processes for organizational use. [16] in their review of the work by [17] note that Activity Theory is an analytical framework in understanding computer-based artefacts as instruments for work activities and materials for systems design for organizations. [16] also notes that Activity Theory is a philosophical and cross-disciplinary framework for studying different forms of human practices as development processes, with both individual and social levels interlinked at the same time in reference to work by [18].

The salient features of the two perspectives imply that the ERP should address both the interactions between the human activities and the elements that form the ERP (the artefacts). The social context addressed by the Activity Theory is centric to the understanding of the key human practices that are to be considered in the implementation of the ERPs. [19] describes these human practices as a community of practices. These communities of practices will inform the artefacts that are to be used in the design and actualization of the ERP. Thus systems should not imposed on users but should be negotiated implementations so that the artefacts are derived from the activities of the community, that is the users.

Since the activity theory emphasizes on work-oriented and participatory approaches to information systems development designs, we draw our framework for the ERPs implementation by not creating a method or methodology, but rather we collect usable methods under the activityphilosophical approach by creating an activity-philosophical model. This in effect will give individual institutions an opportunity to apply the proposed framework of designing ERPs that take care of the prevailing circumstances and hence eliminate the potential user resistance to ERP implementation. 
Activity theory underpins the need to have an all inclusive approach in developing an information system. [20] note that there is need for an analytical model for work-oriented information system design that considers the requirement that what people are doing in their everyday tasks and duties should have an opportunity to make an impact on the prospective information systems an organization may be implementing. [20] further propose that when designing an information system from the worker's perspective, the following be considered: start with the work activity as a systemic entity; see the technology, including computer-based technology, as a tool to facilitate work, embedded in the work system; take into account both collective and individual aspects of work; and study the work systems in their organizational context.

The analytical model for the ERP implementation must be applicable to both technological development by software and information system professionals and the development of work practice itself by the workers.

\section{Proposed Framework for the ERP Implementation}

The proposed framework is anchored on the understanding that ERP implementation for any organization should take into consideration organizational culture and structure. Organizational culture explains people's ways of behaving as exemplified by the organizational structure which includes the rules, programmes and operating procedures. The organizational structure equally defines the practices as indicated by the Activity Theory; which defines the processes through the practices. There is, therefore, need to direct the ERP implementation to the information processing needs (IPN) and information processing capability (IPC). This is to ensure the organization attains a fit between the IPN and IPC for successful system implementation process.

[3] indicate that ERP systems are a class of information processing mechanisms aimed at reducing uncertainty by either providing compatibility among stand-alone systems or fostering exchange of information between the departments within the hierarchical structure. Through OIPT, the assessment of organizational uncertainties in relation to the structure is made possible. For instance, if the information-processing capacity of the existing structure does not support the information-processing requirements (explained by OIPT); employees can refer the uncertainties to upper levels of the hierarchy (structure) for decision-making or further direction. This is so because in any hierarchy, it is expected that the higher levels of the organization have more access to detailed and organization-wide information for decision making than the lower levels, who may not be decision makers.

Uncertainty is associated with insufficient information, leading to inability to confidently predict probabilities of how environments will affect the success or failure of a decision-making task. For instance, the information processing needs of an organization are shaped by the relationship between organizational culture and its structures. Therefore, if the information-processing capacity of the existing organizational structure as identified by OIPT is not high enough to accommodate the information-processing requirements, there is an escalation of uncertainties by the employees to upper levels of the structure. An organization is assumed to be effective if there is a fit between information processing capacity and information processing requirements of its environment [21]. This gives credence to the need for organizations to implement ERPs that accommodate the organizational structure and culture so as to reduce uncertainties. The net effect will be improved information processing capabilities of the ERPs which will address the increased information processing requirements across departments. 
[22] developed a model (Fig. 1) that attempts to address the uncertainty issues associated with the ERP implementations. In the model, they offer explanations on the interaction between organizational culture and organizational structure. In the model, a close relationship is established between organizational structure and culture whereby the organizational structure modifies organizational culture while organizational culture determines the organizational structure. By understanding the organization structure the interdependencies and differentiation characteristics cannot fully be established. We thus propose a modification to the conceptual model presented by [22] to include the OIPT and activity theory concepts as presented in the model Fig. 2.

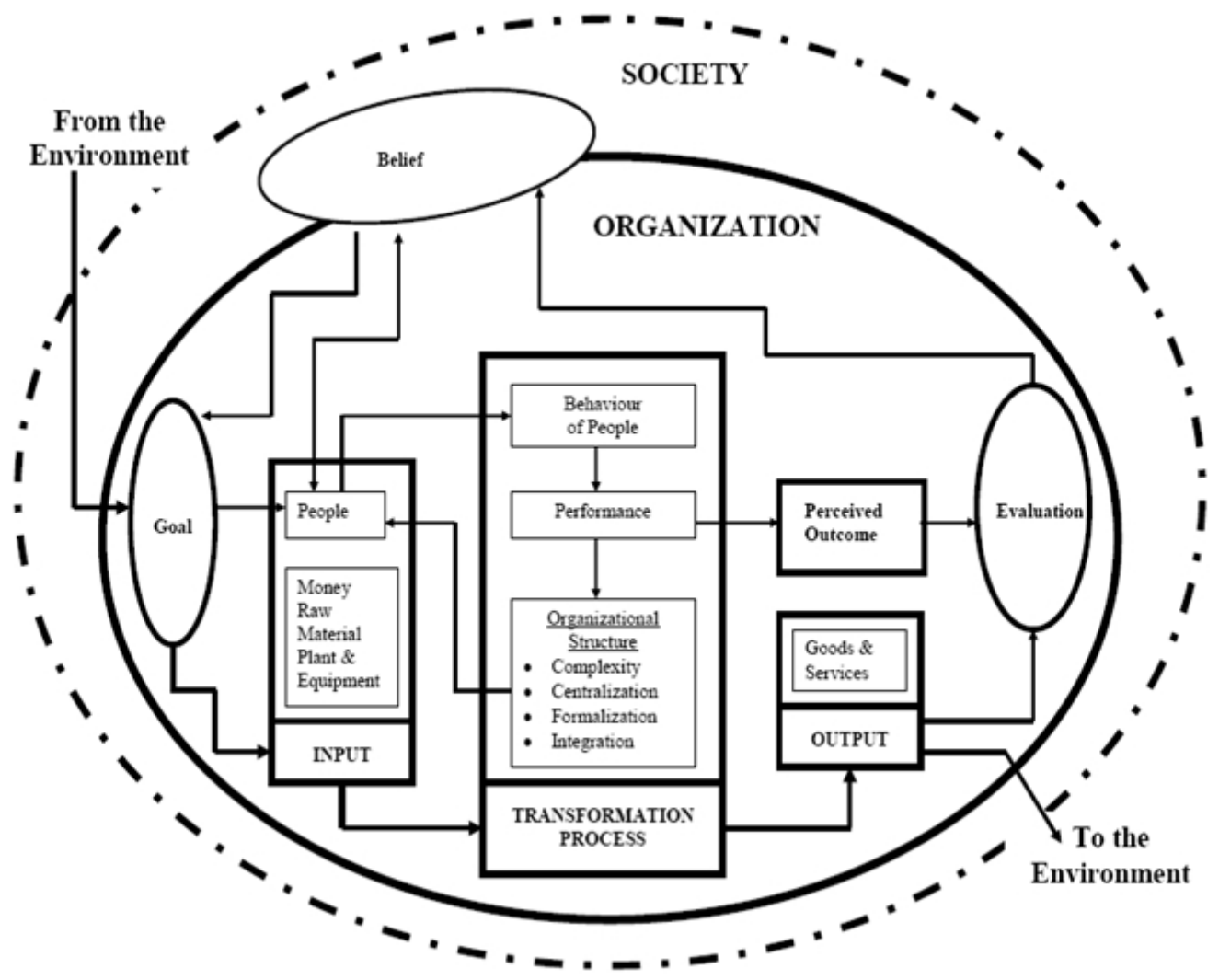

Fig. 1. A conceptual model on the interaction of organizational culture and organizational structure from [22]

As indicated in section 3 above, when an ERP is not a good fit for an organizational unit's unique business processes, processing capability is likely be compromised and when differentiation between units is greater, it is unlikely that an ERP which standardizes processes between units will meet all the unit's needs equally well. Organizational managers therefore need to appreciate the information processing needs of their businesses and measure against the capabilities of the ERP solution to be implemented. 


\subsection{Modified Framework for Successful Enterprise Systems Implementation}

Below we provide a modified framework/ model based on the model developed by Li, Fellows and Liu [22].

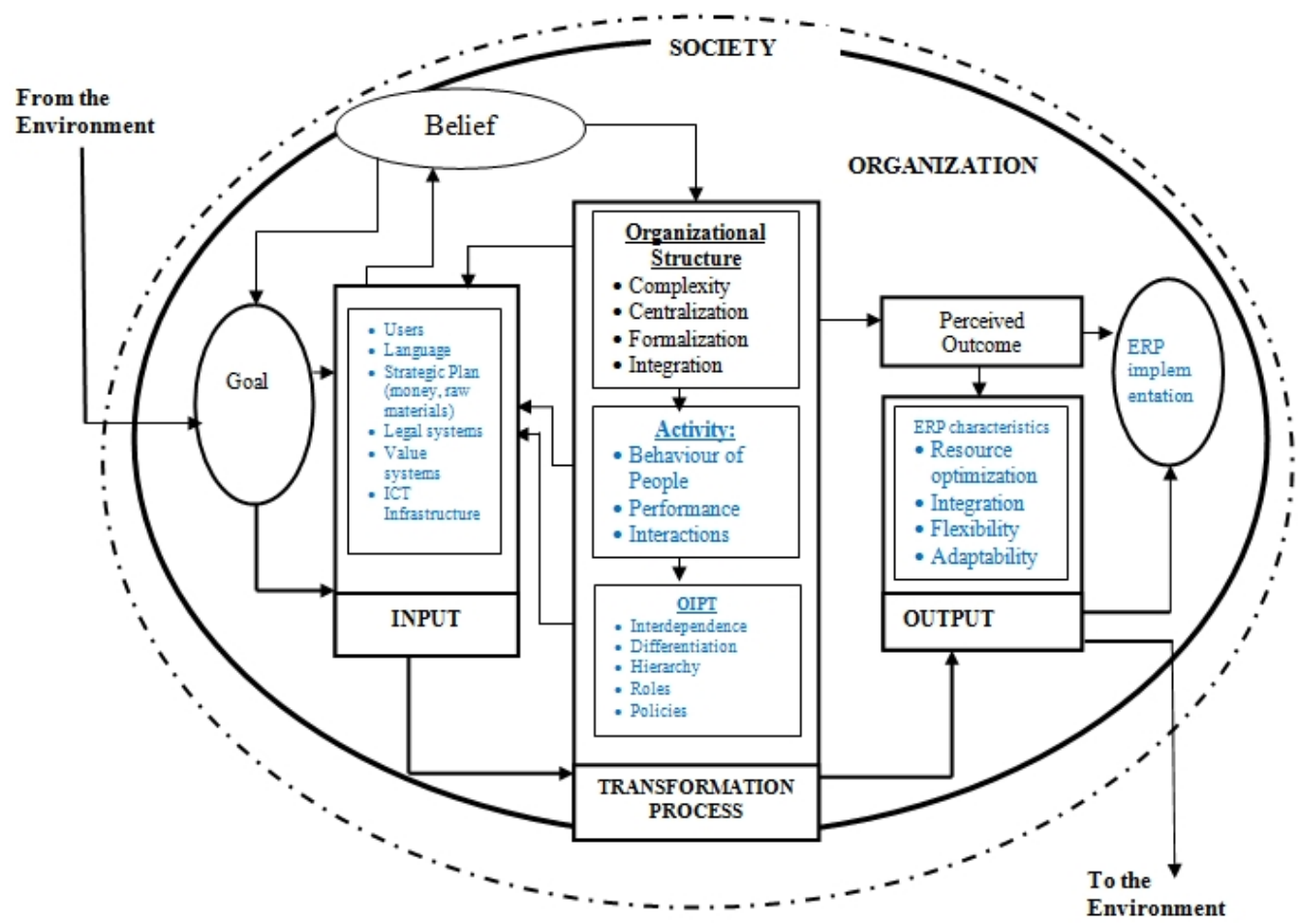

Fig. 2. Modified [22] framework for successful implementation of ERPs

From the figure above, inputs to the ERP system selection for implementation include policies of the institution, the strategic plan, ICT action plan and ICT infrastructure that may already be in use. In the transformation process, the OIPT and activity theory concepts come to play. Activity theory influences the behavior of people, their performance of work, and their interactions in system implementation process. On the other hand, OIPT concepts influence the interdependence of the systems and users as well as the differentiation. In the end the OIPT and activity theory impact on the output shown here as desirable characteristics of ERPs. These characteristics include resource optimization, system integration, flexibility, and adaptability.

The integration of OIPT in ERP implementation can significantly modify the organizational structure to form a new culture, especially where a standard solution like ERP is implemented and staffs are directly involved in the system implementation which effectively reduces the uncertainties associated with the ERP implementation. ERP implementation can bring about new ways of working that can equally lead to modification of the structure, inevitably leading to a flat organization structure as opposed to a tall hierarchical structure. This is because the ERP allows workers to access information stored in large databases that cuts across departments. 


\section{Application of the Proposed ERP Implementation Framework in Universities}

For purposes of informing the practice of systems implementation, the OIPT helps bring forth the needs to ensure capabilities of systems being implemented are well appreciated by the implementers and that the user needs are fully addressed by the solutions proposed. To illustrate on the applicability of the proposed model, a University set-up is considered as it presents an environment in which the various components of the OIPT and Activity Theory are easily identifiable.

[23] and [24] note that an ERP for a university should be implemented on the strength of the modules in it that should facilitate information processing covering students administration (admissions, examinations, fees management, course materials management); staff management (human resources functions, welfare) and resources management (finances, motor vehicles, classrooms, and related facilities). [25] points out that a good ERP for the university set-up should facilitate easy and quick access to students and staff records and should facilitate administration to serve both students and staff efficiently.

In applying the proposed model in the university as pointed out by [25], it is expected that organizational culture, structure, community of practices and existing ICT infrastructure will form important input to the process of procuring and implementing of an organizational ERP. The organization structure, activities to be supported by the system, and the organizational information processing needs form the basis for selection of the ERP. If the process is well managed, then the implementation will be effective and the ERP will lead to important outcomes including resource optimization, integration of business processes, greater flexibility in operations, and optimal adaptability of the business to the operating environment.

In a university, the application of the model will assist in the identification and alignment of the various interplaying variables as indicated by [23] and [24]. For instance, the use of OIPT will assist in the identification of the components of ERPs that are in tandem with the culture and structure of the organization. Some of the components like system values, language within the institution, the legal systems, meaning attached to every action which are critical for system implementation are easily identified. These identified cultural components by the OIPT will define the inputs that will be taken considered by the ERP. By applying the Activity Theory the activities that influence the processes based on the cultural and structural components as formulated by the OIPT is established. Activities like student administration, staff and resource management are aligned with beliefs of the recipient and a hybrid output (processes and input) that represents the desire of all parties in achieved.

Through the application of the proposed model in ERP implementation the users of the ERP will identify with the system through the identified inputs that reflect on their beliefs. The process alignment with the input which occurs through the Activity Theory will further enhance harmony between the users and the systems.

\section{Conclusion and Recommendation}

Information processing requirements and interactions of the various characteristics of an 
organization are critical components for systems to be implemented successively in any organization. By the application of the OIPT the ERP designers and implementers are in a position to establish the organization requirements to be addressed by the system. The activity theory on the other hand is used to establish the work-oriented and participatory approaches to information systems development designs, so as to bring into the system inclusiveness and hence minimize the chances of ERPs implementation resistance by workers.

By applying the proposed model it is anticipated that a balance between input and process while having the user as the central element will promote acceptance of the ERP implementation. Whereas the paper has looked at culture and structure at organizational level, we consider that current organizational models have departmental cultures and structures that contribute to organization-wide processes. Further analysis of the effects of departmental cultural factors on the unit/departmental processes needs to be investigated on how they affect the implementation at the departmental level.

\section{Competing Interests}

Authors have declared that no competing interests exist.

\section{References}

[1] Chen C, Chou SW. The environmental factors and conducted factors that influence the ERP (enterprise resource planning) benefits. Pacific Asia Conference on Information Systems (PACIS): PACIS 2009 Proceedings Association for Information Systems. Accessed on 10 May 2013.

Available: http://www.pacis-net.org/file/2009/[12].

[2] Chou SW, Chang YU. (2008). The implementation factors that influence the ERP (enterprise resource planning) benefits. Elsevier Science Publishers: Decision Support Systems, Volume 46 Issue 1, December 2008; 149-157.

[3] Gattiker TF, Goodhue DL. What happens after ERP implementation: Understanding the impact of inter-dependence and differentiation on plant-level outcomes. MIS Quarterly. 2005;29(3): 559-585.

[4] Premkumar G, Ramamurthy K, Saunders, CS. Information Processing View of Fit in the Context of Interorganizational Relationships. 2005; Journal of Management Information Systems 22: 257-294.

[5] Karimi, J, Somers, TM, Gupta YP. Impact of Environmental Uncertainty and Task Characteristics on User Satisfaction with Data. Information Systems Research, 2004;15(2):175-193.

[6] Daft RL, Lengel RH. Organizational information requirements, media richness and structural design. Management Science. 1986;32:554-571. 
[7] Soh C, Kien SS, Tay-Yap J. Cultural Fits and Misfits: Is ERP a Universal Solution. Communications of the ACM. 2000;43(4):47-51.

[8] Copper RB, Zmud RW. Information technology implementation research: a technological diffusion approach. Management Science, 1990;36(2):123-139.

[9] Hong KK, Kim YG. The Critical Success Factors for ERP Implementation: An Organizational Fit Perspective. Information and Management. 2002;40:25-40.

[10] Morton NA, Qing H. Implication of the fit between organizational structure and ERP: A structural contingency theory perspective. International Journal of Information Management. 2008;28:391-402.

[11] Gattiker TF, Goodhue DL. Understanding the local-level costs and benefits of ERP through organizational information processing theory. Information \& Management. 2004;41:431-443.

[12] Jones BD, Boushey G, Workman S. Behavioral Rationality and the Policy Processes: Toward A New Model of Organizational Information Processing. Accessed on 10 April 2013.

Available: http://www.princeton.edu/ smeunier/JonesBousheyWorkman.pdf.

[13] Wybo MD, Goodhue, DL. Using interdependence as a predictor of data standards: theoretical and measurement issues. Information and Management. 1995;29:317328.

[14] Cooke DP, Peterson WJ. SAP implementation strategies and results.; Conference Research Report (1998). Accessed on 4 May 2013; 1998. Available: https://www.wlu.ca/documents/17395/ERP CRM SCM paper.pdf

[15] Otieno JO. Enterprise Resource Planning Systems Implementation and Upgrade: A Kenyan Study. Unpublished PhD thesis, Middlesex University, UK; 2010.

[16] Mursu A, Luukkonen I, Toiranen M, Korpela M. Activity Theory in Information Systems Research and Practice: Theoretical Underpinning for an Information System Development Model. IR Information Research. 2007;12(3).

[17] Bødker S. Activity theory as a challenge to systems design, in HE. Nissen, H Klein and R Hirscheim (eds.), Information systems research: contemporary approaches and emergent traditions. Amsterdam: Elsevier. 1991;551-564.

[18] Kuutti K. Activity theory as a potential framework for human-computer interaction research, in Nardi (ed.), Context and consciousness: activity theory and humanComputer interaction. Cambridge: MIT Press. 1996;17-44. 
[19] Wenger E. Communities of Practice: Learning, meaning and identity. Cambridge University Press; 1999.

[20] Korpela M, Mursu A, Soriyan A, Eerola A, Häkkinen H, Toivanen MIS. Research and development by activity analysis and development - dead horse or the next wave? In Kaplan B, Truex III D, Wastell D, Wood-Harper AT, DeGross J.I. (eds.), Information systems research - relevant theory and informed practice. Boston: Kluwer Academic. 2004;453-471.

[21] Wald A. A Micro-level approach to Organizational Information Processing. Schmalenbach Business Review. 61 July 2009;270-289.

[22] Li YIH, Fellows R, Liu AMM. Application of Information Processing Theory on the Inter-Relationship of Organizational Culture and Organizational Structure. International Conference on Multi-National Construction Projects- Securing high Performance through Cultural awareness and Dispute Avoidance. Shanghai, China; 2008.

[23] Cornelius ET. Seven Steps in the ERP Process: An Overview of the Higher Ed ERP Journey. Accessed $23^{\text {rd }}$ February 2013; 2007.

Available: http://www.collegiateproject.com.

[24] Pollock N, Cornford J. ERP systems and the university as a "unique" organization. Information Technology \& People. 2004;17(1).

[25] Rico DF. ERP in Higher Education. Accessed 23 March 2013; 2004. Available: http:// www.davidfrico.com.

(C) 2014 Nyandiere et al.; This is an Open Access article distributed under the terms of the Creative Commons Attribution License (http://creativecommons.org/licenses/by/3.0), which permits unrestricted use, distribution, and reproduction in any medium, provided the original work is properly cited. 